\title{
Combination of natural teeth and osseointegrated implants as prosthesis abutments in a posterior cantilever bridge
}

\author{
Michael Josef Kridanto Kamadjaja \\ Department of Prosthodontic \\ Faculty of Dentistry, Airlangga University
}

Surabaya - Indonesia

\begin{abstract}
Dental implants have been used for several decades. Patients of all ages have chosen dental implants to replace a single tooth or several teeth or to support partial or full dentures. This paper reports two cases of patients treated with dental implant as alternative to replace the missing teeth and connected with natural tooth as abutments in a fixed restoration with distal cantilever bridge. The underlining reasons that we decided to make such kind fixed prostheses are because of clinically imposible to put the implant on certain area and the patients asked for prostheses as optimum as possible, so the mastication function could return to the homeostasis condition. The benefit of these treatments are that prostheses could be made as optimum as possible with a more economic price, so the patients feel quite satisfied. The result shows that a few years after the treatments finished there is no any disadvantageous effect of connecting teeth to implants as abutments in fixed partial dentures and there is no sign of a harmful effect to the opposing teeth either.
\end{abstract}

Key words: connecting implant to natural tooth, osseointegration

Correspondence: Michael Josef Kridanto Kamadjaja, c/o: Departemen Prostodonsia, Fakultas Kedokteran Gigi Universitas Airlangga. Jln. Mayjend. Prof. Dr. Moestopo no. 47 Surabaya 60132, Indonesia. E-mail: josef_310563@ yahoo.com

\section{INTRODUCTION}

A dental implant is an artificial tooth root that places into your jaw to hold a replacement tooth or bridge. Dental implants are an ideal option for people in good general oral health who have lost a tooth or teeth due to periodontal disease, an injury, or some other reason. Dental implants are so natural-looking and feeling, you may forget you ever lost a tooth Dental implants are teeth that can look and feel just like your own! Under proper conditions, such as good and diligent patient maintenance, implants can last a lifetime. Long-term studies continue to show improving success rates for implants. ${ }^{1}$

Implant treatment generally is a three-part process that takes several months. In the first step, the dentist surgically places the implant in the jaw, with the top of the implant slightly above the top of the bone. A screw is inserted into the implant to prevent gum tissue and other debris from entering.

The gum then is secured over the implant. The implant will remain covered for approximately three to six months while it fuses with the bone, a process called "osseointegration". There may be some swelling, tenderness or both for a few days after the surgery, so pain medication usually is prescribed to alleviate the discomfort. A diet of soft foods, cold foods and warm soup often is recommended during the healing process.

In the second step, the implant is uncovered and the dentist attaches an extension, called a post, to the implant. The gum tissue is allowed to heal around the post. Some implants require a second surgical procedure in which a post is attached to connect the replacement teeth. With other implants, the implant and post are a single unit placed in the mouth during the initial surgery. Once healed, the implant and post can serve as the foundation for the new tooth. In the third and final step, the dentist makes a crown, which has a size, shape, colour and fit that will blend with your other teeth. Once completed, the crown is attached to the implant post. $^{2}$

Endosteal implant is the most commonly used type of implant. The various types include screws, cylinders or blades surgically placed into the jawbone. Each implant holds one or more prosthetic teeth. Root form implants are the closest is shape and size to the natural tooth root. They are commonly used in wide, deep bone to provide a base for replacement of one, several or a complete arch of teeth. This type of implant is generally used as an alternative for patients with bridges or removable dentures. ${ }^{1,2}$

Some cases were done by connecting an implant with a natural tooth as abutments in a fixed restoration. ${ }^{3,4,5}$ This opinion is still remain pro and contra between the dentists. Some of them are agree of connecting implant to natural tooth, but the others are still disagree. The purpose of this case report is to show the connection of implant to natural tooth as abutments in a fixed restoration with posterior cantilever pontic for certain conditions.

\section{CASES}

Two cases of patients treated with dental implant as alternative to replace the missing teeth are reported. Those 
two patients are A-48-year old female patient presented in case 1 and A-63-year old male patient presented in case 2. Both patients asked for dental implant as the refused for removable prostheses after their teeth being extracted. In both cases the author used different type of implant abutment, 2-pieces implant abutment for case no 1 and 1 -piece abutment for case no. 2. In case no 1 we used Oraltronic Implant and in case no. 2 we used Q-Implant (Trinon). Both of them were osseointegrated implant, where we waited four to six months before complete the procedure with the placement of a porcelain bridge. Informed consent had been aproved by the patients before the treatments began.

\section{CASE MANAGEMENT}

Case 1: A-48-year old female patient came to the dental practice with chief complaints of pain in chewing at the left lower side. It had been starting since 3 days ago and she had not taken any medicine yet. Clinical and panoramic examinations revealed that tooth 37 was broken and should be extracted (Figure 1). The patient did not want use a removable prostheses and she asked for dental implant treatment and bridge denture rehabilitation after the tooth being extracted.

Porcelain bridge 35, 36, 37 was separated and tooth 37 was extracted, a deep curettage was done to clean the socket from cyst and the gums closed with several stiches. One week after that the stiches were opened and the patient didn't feel anything. Patient came back five months after the extraction and brought panoramic photo also. The region $37 \mathrm{did}$ not

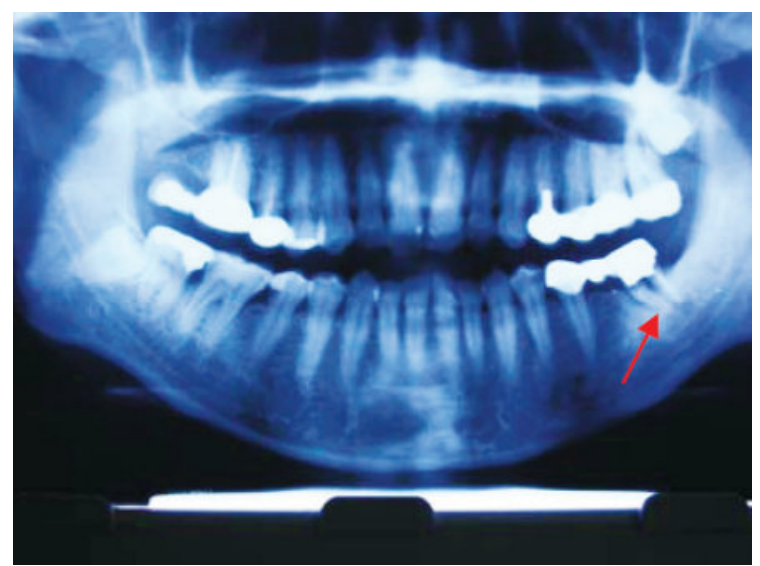

Figure 1. Panoramic radiography view: tooth 37 was broken.

look to have enough density (Figure 2a \& c), so we decided to put the implant fixture in region 36. After application of anesthetic, we did several of incisions and bone preparations before the implant being placed. A pitt -easy bio-oss implant from Oraltronics with $3.75 \mathrm{~mm}$ diameter and $12 \mathrm{~mm}$ length was inserted in region 36 (Figure $2 \mathrm{~b} \& \mathrm{~d}$ ) and the gums are closed with several stitches. The patient was covered with antibiotic, anti inflammation and analgesic drug during the healing process. ${ }^{2}$ Continuance of normal oral hygiene with chlorhexidine mouth rinses would help to prevent wound infection. Up to three weeks showed no clinical symptoms and inflammatory process. Tooth 34 that suffered from gangraen pulpae was treated with root canal treatment and strengthened with metal post in it. We ordered the patient to come back in the next 4 months.
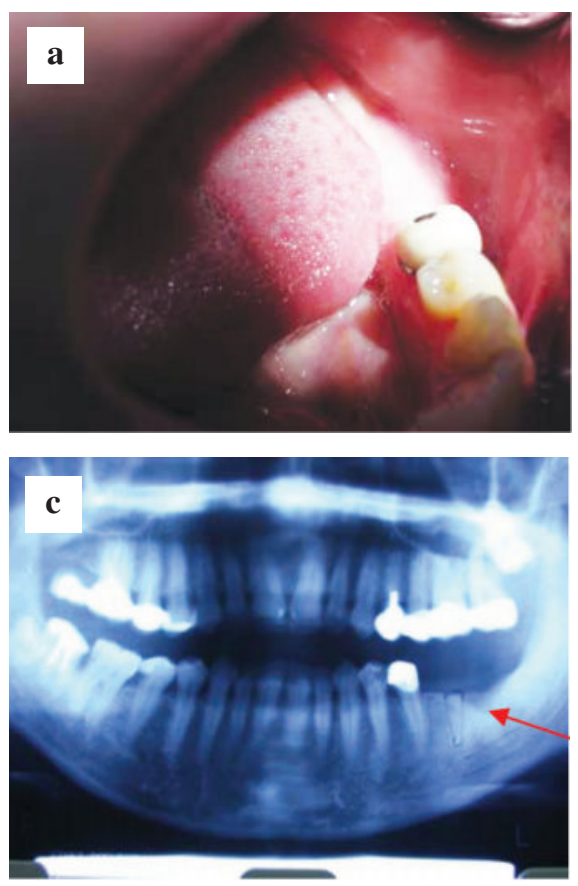
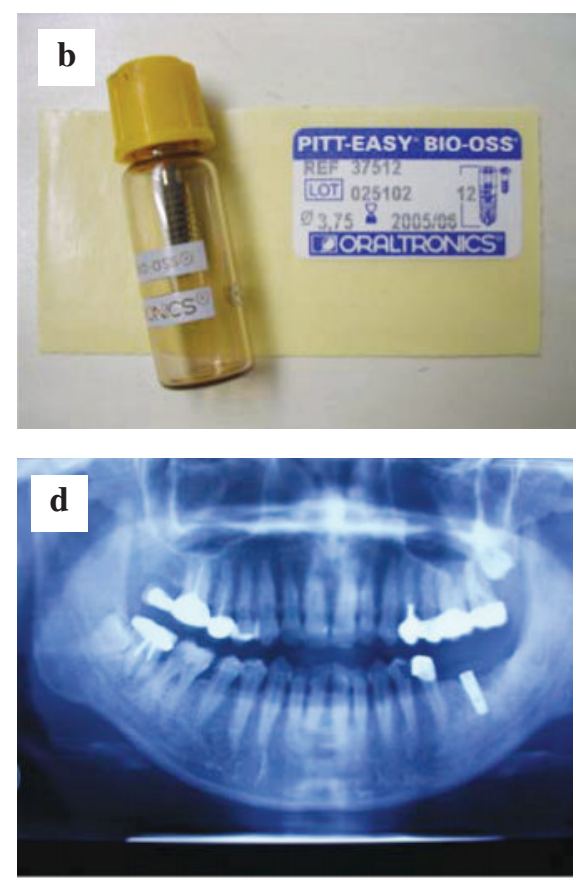

Figure 2. a) Intraoral condition in region 37 before implant placement; b) A pitt -easy bio-oss implant from Oraltronics with $3.75 \mathrm{~mm}$ diameter and $12 \mathrm{~mm}$ length; c) Region 37 does not look have enough density; d) Implant fixture was inserted in region 36. 
When the patient came back, we opened the cover screw and we changed with healing screw (Figure 3a). A week after that we changed the healing screw with the abutment post and a porcelain cantilever bridge was done from teeth 34, 35, 36, 37 (Figure 3b-f). Panoramic radiograph six months after the treatment finished showed that there was nothing wrong with the treatment (Figure $3 \mathrm{~g}$ ). Up till now it has been 4 years after the treatment finished and there was nothing wrong with the treatment.
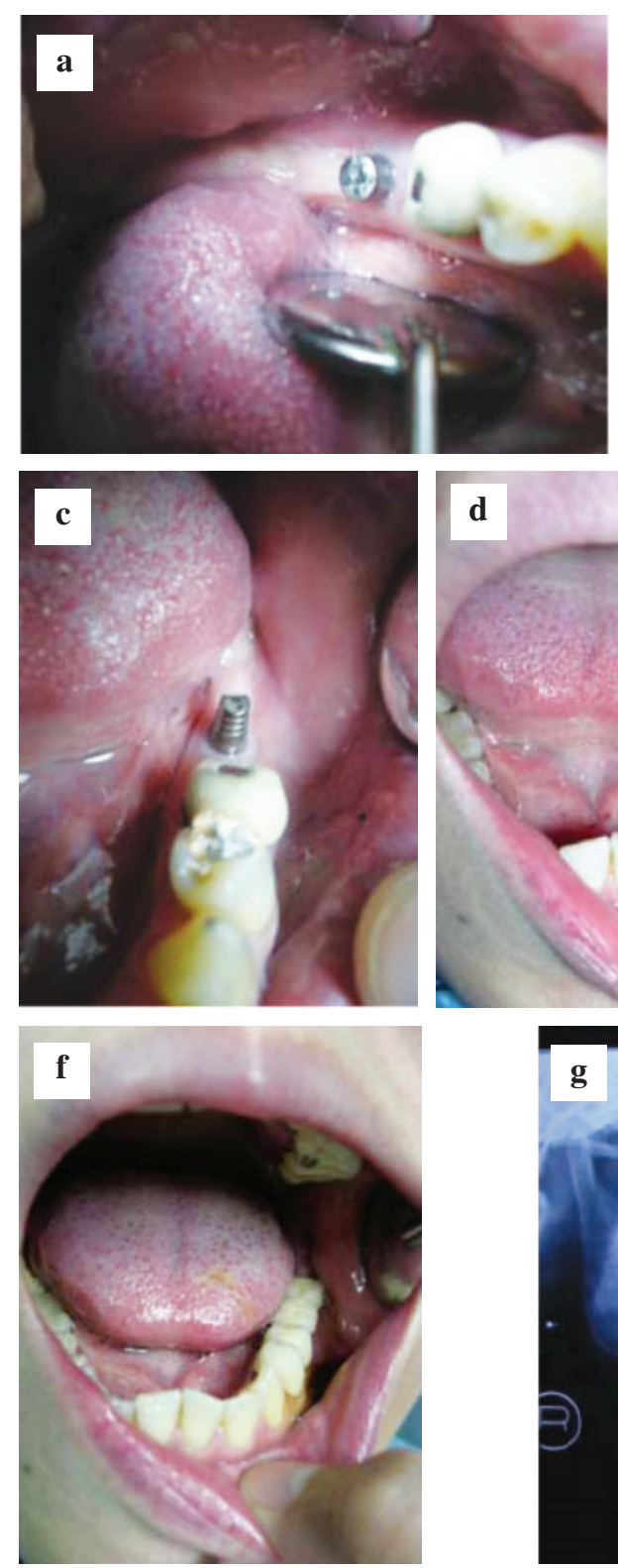
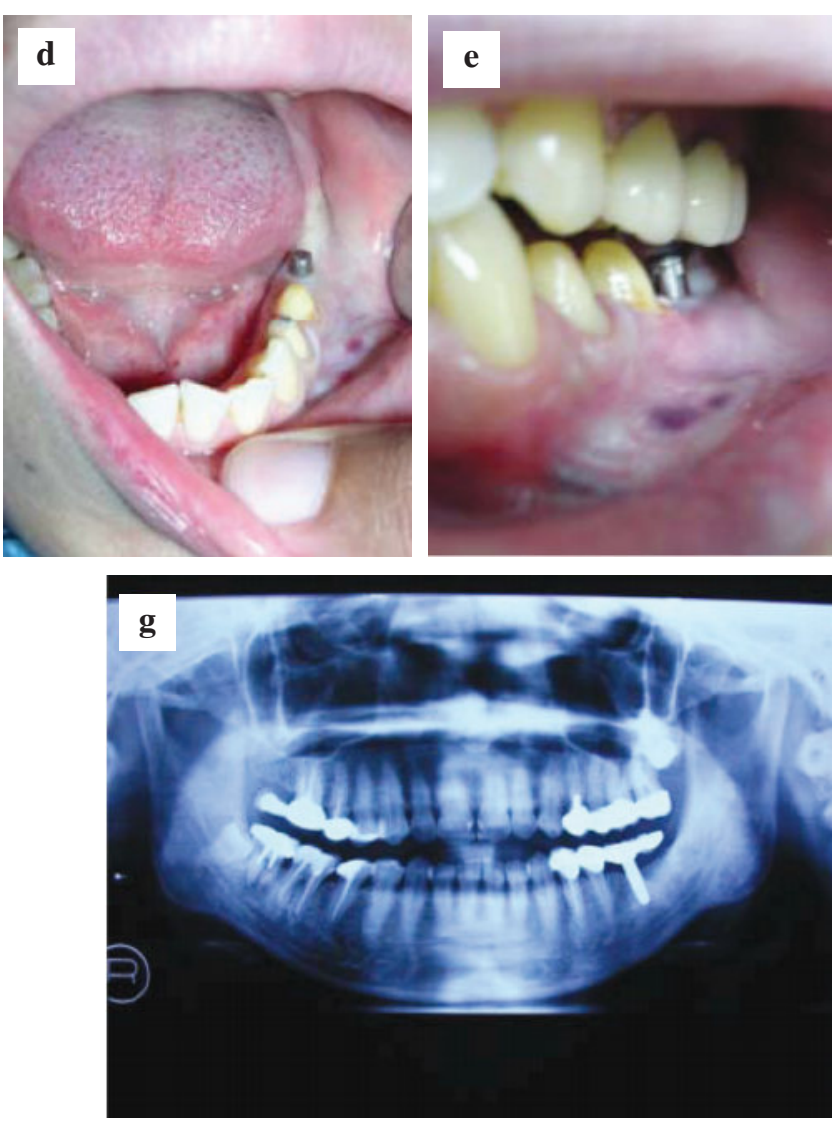

Figure 3. a) Healing screw was attached to the implant fixture; b) Healing screw was opened; c) Abutment post was attached to the implant fixture; d) Teeth 34, 35 and abutment post were prepared; e) Their relation to the opposing teeth; f) Cantilever porcelain bridge from 34, 35, 36, 37 was inserted; g) Panoramic radiography view: Cantilever porcelain bridge from 34, $35,36,37$.

Case 2: A-66-year old male patient came to the dental practice with chief complaint of difficulty in chewing at the left side. Clinical examination showed that teeth 24 till 27 had been extracted (Figure $4 \mathrm{a} \& \mathrm{~b}$ ). The patient had a removable partial denture, but he did not feel comfortable with it, so he asked for a fix artificial dentures. He agreed for having dental implant treatment and bridge denture rehabilitation
After application of anesthetic by using a tissue punch with $3 \mathrm{~mm}$ diameter we made a hole on the gum. Pilot drill was used to make an optimal implant direction until $12 \mathrm{~mm}$ depth was reached and continued with shaping drill with selected diameter and length. The implant was gently taken from the sterile cover and inserted into the prepared bone cavern with the insertion wrench. It might be helpful to use the hand wheel or hand wrench. One Q-Implant from Trinon 
with $3.5 \mathrm{~mm}$ diameter, standard neck heights $(4 \mathrm{~mm})$ and $12 \mathrm{~mm}$ length were inserted in region 25 (Figure $4 \mathrm{c}$ ). The Q-Implant were constructed with a self-cutting thread and inserted by using minimal force could achieved a high primary stability. ${ }^{6}$ Tooth 23 was prepared and temporary bridge from 23, 24, 25 was placed. The patient was covered with antibiotic, anti inflammation and analgesic drug during the healing process. Clinical symptoms as pain, implants
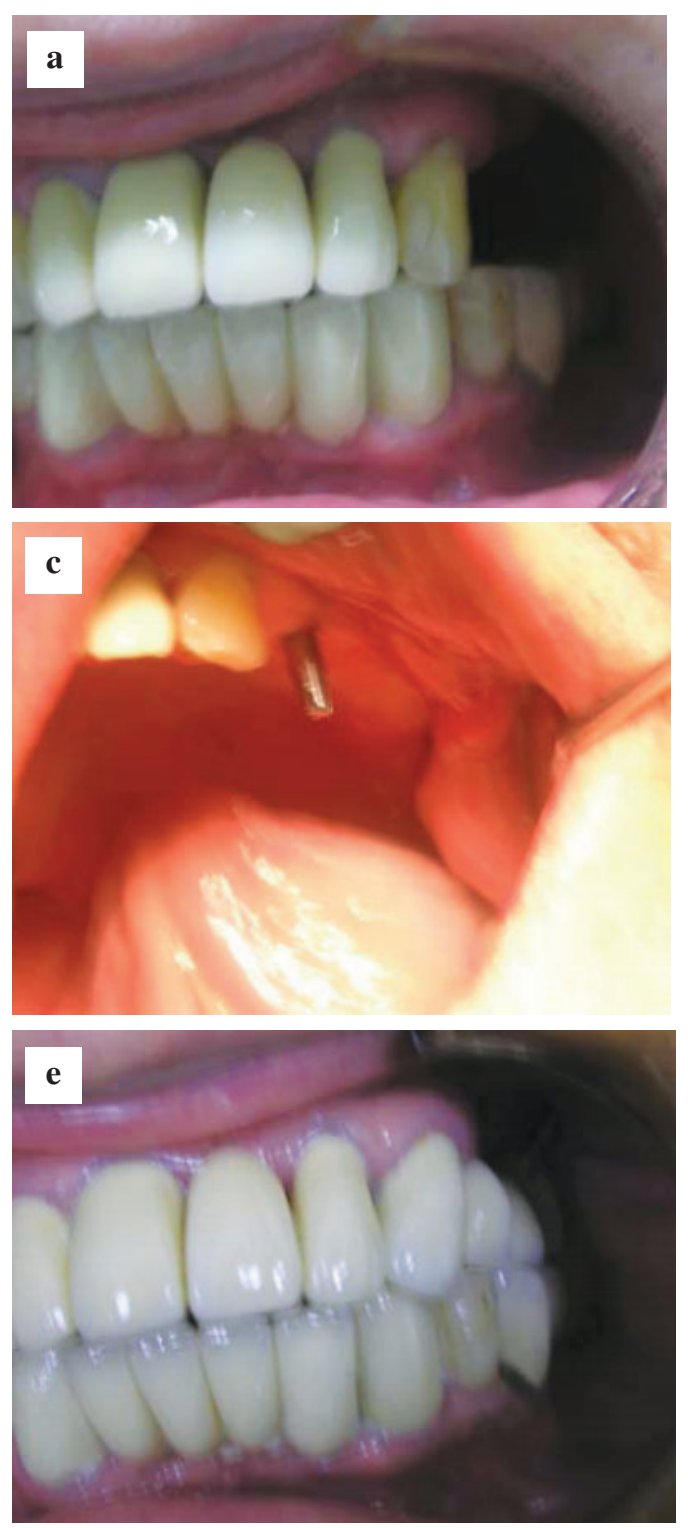

mobility and sign of inflammatory process were not presented. Chlorhexidine mouth rinses was also prescribed for preventing wound infection and maintaining the normal oral hygiene. ${ }^{2}$ During the osseointegration period, the patient did not allow to use the left side of his teeth for chewing food. Four months after the implant placement, cantilever porcelain bridge was made from teeth 23,24 , 25, 26 (Figure 4d-f). We ordered the patient to come back
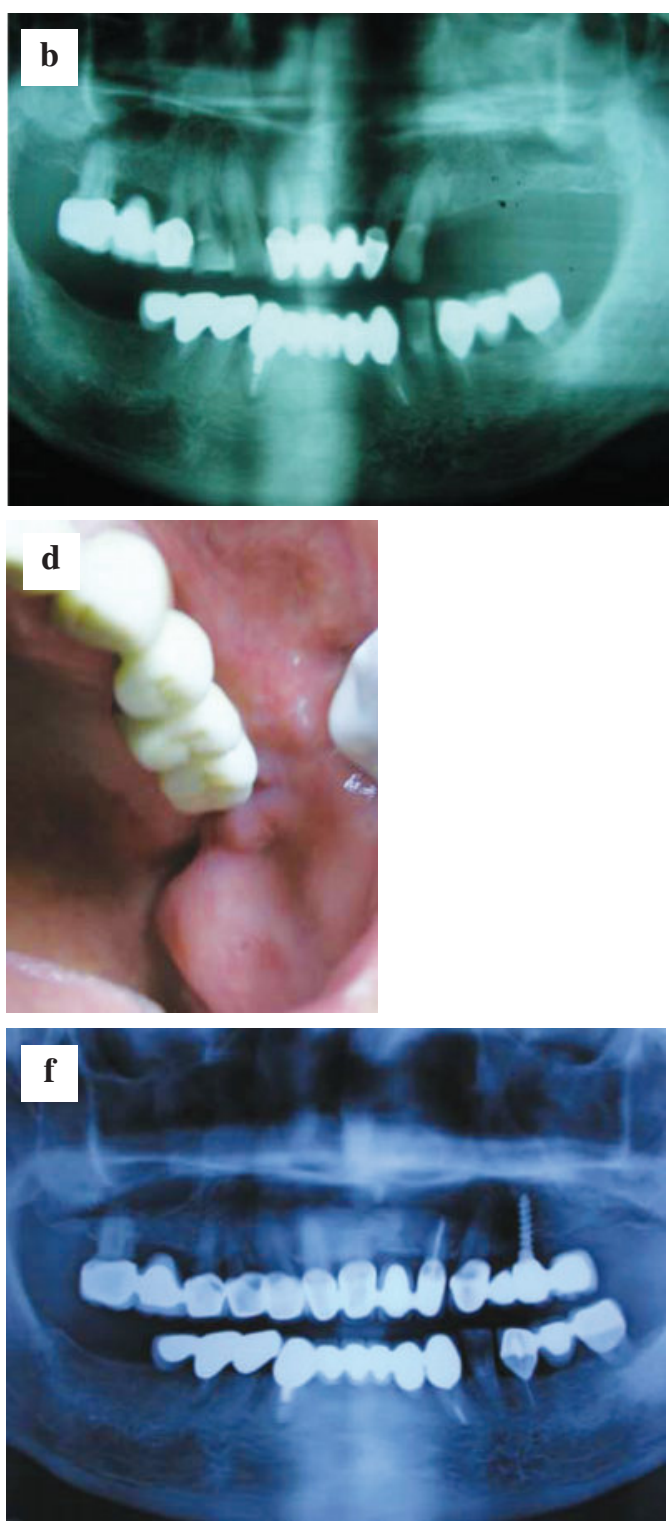

Figure 4. a) Intraoral condition before implant placement; b) Panoramic radiographic view : region 24, 25, 26, 27; c) Q-Implant from Trinon with 3,5 $\mathrm{mm}$ diameter, standard neck heights $(4 \mathrm{~mm})$ and $12 \mathrm{~mm}$ length were inserted in region 25; d) Cantilever porcelain bridge in region 23, 24, 25, 26 was inserted; e) Interocclusal relation after the porcelain bridge was inserted; f) Panoramic radiographic view: Cantilever porcelain bridge from 23, 24, 25, 26.

every six months for routine check up and up till now it has been two years after the treatment finished and there was nothing wrong with the treatment.

\section{DISCUSSION}

Two cases of dental implant placement were reported. In case 1 we used two phase concept of implant placement which the abutment post was engaged by using screw 
type and in case 2 we used one phase concept of implant placement. In case 1, which we used two phase concept of implant placement, we needed more implant process than case 2, which used one phase concept of implant placement.

Both cases were delayed loading which waited 4 to 8 months before providing the tooth restoration. In case 1 the implant placement was done by making several incisions, but in case 2 we did it without making any incision (flapless). Implant placement with flapless could reduced the bleeding of the blood and wound healing could be achieved in a short time, but if you did not sure about the bone condition you better did it with any flap, because we could do bone management better than flapless.

Dental implants are among the most successful procedures in dentistry. There is no guarantee that an implant procedure will be successful, but studies have shown a five-year success rate of $95 \%$ for lower jaw implants and $90 \%$ for upper jaw implants. The success rate for upper jaw implants is slightly lower because the upper jaw (especially the posterior section) is less dense than the lower jaw, making successful implantation and osseointegration potentially more difficult to achieve. Lower posterior implantation has the highest success rate for all dental implants. ${ }^{7}$

Two cases of patients treated with dental implant as alternative to replace the missing teeth that connected with natural teeth are reported. Connecting dental implants and natural teeth are still become pro and contra between the dentists. The reason why dentist did not like splinting implants with natural teeth is for one major reason. Dentist believed that because the natural tooth has a periodontal membrane surrounding it with a certain degree of mobility, although this movement is very small. Many dentist believe because a dental implant is osseo-integrated and there is no periodontal membrane around it, so the dental implant will fail because the movement of the natural tooth will cause the implant to move slightly. It is speculated that this movement will cause the dental implant to fail. ${ }^{8}$

According to purely theoretical considerations, the splinting of osseointegrated (functionally ankylosed) implants to natural teeth that are suspended by a periodontal membrane with a certain degree of mobility is not rational. Because the two types of attachment are basically different it is possible that while functioning, the involved implant abutment is the primary recipient of the load, comparable to a cantilever bridge abutment. Whether or not this has any effect on the prognosis is still unclear at the present time. ${ }^{3,4,5}$ In general, it seems advisable to avoid connecting natural teeth to implant abutments whenever possible. Naert et al. ${ }^{9}$ said that more bone is lost around implants which are rigidly connected to teeth than freestanding ones. Over the period from 0 to 15 years, there was significantly more marginal bone loss $(0.7 \mathrm{~mm})$ in tooth-implant connected versus freestanding prostheses.

Kindberg et al. ${ }^{10}$ confirms that treatments with periodontal healthy teeth and implants splinted together in rigid one-piece superstructures show excellent longterm follow-up results. Srinivasan and Padmanabhan ${ }^{11}$ concluded that it is indeed beneficial to connect natural tooth to implants in a fixed partial restoration and that the type of connector advocated is a rigid one. Also conclusive is the fact that periodontally compromised teeth can be integrated in the restoration in combination with an implant as a conservative treatment option. The use of non rigid connectors in any situation may be erroneous.

Takeda ${ }^{12}$ did a 10 year study and presented his findings, "The Harmony of ITI Implants and Natural Teeth". In summary his observations were that "connection distance had a significant effect on changes to teeth, i.e., intrusion or cement washout". This seems to suggest that if you have to connect teeth to implants the distance between the two should be at least $10 \mathrm{~mm}$ or so. I have had no problems when following this rule.

Clinical study by Radnai et al. ${ }^{13}$ found that there is no disadvantageous effect of connecting teeth to implants as abutments by fixed partial dentures was found. There was no sign of a harmful effect of the implant to the opposing teeth either. The tooth-to-implant bridges function in their biological environment without affecting it adversely.

In two cases above, cantilever porcelain bridges were made as fixed restorations because the patients asked that the restoration could reach the optimum distal point, so they could chew the food properly. The underlining reasons that we decided to make a posterior cantilever bridge that supported by tooth-implant abutments are because of clinically imposible to put the implant on certain area and the patients asked for prostheses as optimum as possible, so the mastication function could return to the homeostasis condition. The benefit of this kind of treatment is that prostheses could be made as optimum as possible with a more economic price, so the patients feel quite satisfied and up till now there are not any problems with these two cases. The treatment lacks of these treatments are combining tooth and implant as abutments of prostheses are still debated between dentists and fixed prostheses with cantilever pontic are not preferred by the dentists, because they are afraid that there would be any harmful effect to the abutments.

Distal cantilever pontic, based upon the unfavourable leverage and negative experiences reported in the literature for similarly configured conventional prostheses, this variation should be avoided whenever possible. ${ }^{14}$ The occlusal surface area of the pontic is generally decreased by making the pontic smaller than the original tooth, so the abutments did not receive too much load. The dimensions of the bridge are defined by Ante's Law: "The root surface area of the abutment teeth has to equal or surpass that of the teeth being replaced with pontics". ${ }^{15}$

Jan et al. ${ }^{16}$ from his study failed to demonstrate that the presence of cantilever extensions in an Fixed Partial Dentures (FPD) had an effect on peri-implant bone loss, but smoking had a significant influence on peri-implant bone level change on the FPD level. 
Romeo et $a l .{ }^{17}$ from his study that performed on a sample of 38 partially edentulous patients treated with 49 partial cantilever fixed prostheses and supported by 100 implants said that seven years after loading cantilever prostheses, the overall cumulative implant survival rate (OCSR) was $97 \%$ and the prostheses success rate was $98 \%$. Mesial cantilever prostheses registered a lower success rate $(97.1 \%)$ than distal cantilever prostheses $(100 \%)$. Furthermore, a better prognosis was not observed when the opposite dentition of the prostheses comprised natural teeth, or fixed prostheses on natural teeth, when compared with the cases in which opposite teeth were implant-supported fixed prostheses.

In spite of all that statements, it was concluded that combining implants to natural teeth as abutment of fixed prostheses with cantilever pontic although are still remained pro and contra among the dentists, but that kind of treatment should be considered as a viable prosthetic option. Suppose we should do it, some conditions must be fulfilled, e.g periodontally healthy teeth, rigid connection between tooth and implant, successful implantation and osseointegration could be achieved properly.

\section{REFERENCES}

1. World Centre for Dental Implantology. Copyright 2001. Available at http://www.Implant Types.htm. Accessed December 23, 2007.

2. Floyd P, Palmer R, Barrett V. Treatment planning for implant restorations. British Dental Journal 1999 Sept; 187(6):25.

3. Schroeder A, Sutter F, Buser D, Krekeler G. Oral implantology. Basics, ITI Hollow cylinder system. $2^{\text {nd }}$ ed. New York: Thieme Medical Publisher, Inc; 1996. p. 243-6.
4. Astrand P, Borg K, Gunne J, Olsson M. Combination of natural teeth and osseointegrated implants as prosthesis abutments: a 2-year longitudinal study. Int J Oral Max-Fac Implants 1991; 6:305-12.

5. Gunne J, Astrand P, Ahlen K, Borg H, Olsson M. Implants in partially edentulous patients. A longitudinal study of bridges supported by both implants and natural teeth. Clin Oral Impl Res 1992; 3:49-56.

6. Concept TRINON IMPLANT.htm. Accessed January 12, 2008.

7. Johnstone G. Dental implants. Available at http://Dental Implants -A Complete Consumer Guide.htm. Accessed December 23, 2007.

8. $\mathrm{PD} /$ "connecting-implants-and-natural-teeth ?"/ Available at: www. osseonews.com//\#comment- 4002.htm. Accessed January 10, 2008.

9. Naert IE, Duyck JA, Hosny MM, Quirynen M, van Steenberghe D. Freestanding and tooth-implant connected prostheses in the treatment of partially edentulous patients Part II: An up to 15-years radiographic evaluation. Clin Oral Implants Res 2001 Jun; 12(3):245-51.

10. Kindberg H, Gunne J, Kronström M. Tooth and implant supported prostheses: a Retrospective clinical follow-up to 8 years. Int J Prosthodont 2001 November-December; 14(6):575-81.

11. Srinivascan M, Padmanabhan T. Implant prosthodontic: An in-vitro photoelastic stress analysis. Journal of Indian Prosthodontic Society 2005;

12. Takeda $\mathrm{T}$. The harmony of ITI implants and natural teeth. Available at: www.osseonews.com/Dental Implant Discussion / Dental Implants Connecting Natural Teeth.htm. Accessed January 10, 2008.

13. Radnai M, Fazekas A, Vajdovich I, Kostinek D, Fogorv SZ. Clinical study of tooth-to-implant supported fixed partial dentures. Dental Implant Research 1998 July; 91(7):195-202.

14. Strub JR, Linter H, Marinello CP. The rehabilitation of partially edentulous cases with cantilever bridge bridges: a retrospective study. Int J Periodont Restorat Dent 1989; 9:364.

15. Shillingburg HT, Hobo S, Whitsett LD, Jacobi R, Brackett SE. Fundamentals of fixed prosthodontics. $3^{\text {rd }}$ ed. Quintessence Publishing Co, Inc 1997; 92.

16. Jan W, Zurdo J, Karlsson S, Annika E, Kerstin G, Lindhe J. Bone level change at implant-supported fixed partial dentures with and without cantilever extension after 5 years in function. J of Clin Periodontology December 2004; 31(12):1077-83.

17. Romeo E, Lops D, Margutti E, Ghisolfi M, Chiapasco M, Vogel G. Implant-supported fixed cantilever prostheses in partially edentulous arches. A seven-year prospective study. Clin Oral Implants Res 2003; 14:303-11. 